\title{
Talker continuity and the use of rate information during phonetic perception
}

\author{
KERRY P. GREEN \\ University of Arizona, Tucson, Arizona \\ and \\ ERICA B. STEVENS and PATRICIA K. KUHL \\ University of Washington, Seattle, Washington
}

\begin{abstract}
Research has shown that speaking rate provides an important context for the perception of certain acoustic properties of speech. For example, syllable duration, which varies as a function of speaking rate, has been shown to influence the perception of voice onset time (VOT) for syllableinitial stop consonants. The purpose of the present experiments was to examine the influence of syllable duration when the initial portion of the syllable was produced by one talker and the remainder of the syllable was produced by a different talker. A short-duration and a long-duration $/ \mathrm{bi} /-/ \mathrm{pi} /$ continuum were synthesized with pitch and formant values appropriate to a female talker. When presented to listeners for identification, these stimuli demonstrated the typical effect of syllable duration on the voicing boundary: a shorter VOT boundary for the short stimuli than for the long stimuli. An /i/ vowel, synthesized with pitch and formant values appropriate to a male talker, was added to the end of each of the short tokens, producing a new hybrid continuum. Although the overall syllable duration of the hybrid stimuli equaled the original long stimuli, they produced a VOT boundary similar to that for the short stimuli. In a second experiment, two new /i/ vowels were synthesized. One had a pitch appropriate to a female talker with formant values appropriate to a male talker; the other had a pitch appropriate to a male talker and formants appropriate to a female talker. These vowels were used to create two new hybrid continua. In a third experiment, new hybrid continua were created by using more extreme male formant values. The results of both experiments demonstrated that the hybrid tokens with a change in pitch acted like the short stimuli, whereas the tokens with a change in formants acted like the long stimuli. A fourth experiment demonstrated that listeners could hear a change in talker with both sets of hybrid tokens. These results indicate that continuity of pitch but not formant structure appears to be the critical factor in the calculation of speaking rate within a syllable.
\end{abstract}

During normal conversation, talkers make frequent and extensive changes in their overall speaking rate (Miller, Grosjean, \& Lomanto, 1984). Such changes are accomplished by modifying the frequency and duration of pauses, as well as the overall articulation rate of phonetic segments. Changes in the articulation rate result in a large amount of variation in the temporal characteristics of the speech signal (Miller \& Baer, 1983; Miller, Green, \& Reeves, 1986; Port, 1979; Summerfield, 1975; Volaitis \& Miller, 1992; see Miller, 1981 for review). For example,

This research was supported in part by Research and Training Grant 1 P60 DC-01409 from the National Institute of Deafness and Other Communication Disonders, National Institutes of Health Grant NS-26475 to K.P.G., and National Institutes of Health Grant HD-18286 to P.K.K. A portion of these data were presented at the spring meeting of the Acoustical Society of America, 1989, in Syracuse, NY. The authors would like to thank two anonymous reviewers for their helpful comments. Requests for reprints should be addressed to K. P. Green, Cognitive Science, Psychology Building, Room 312, University of Arizona, Tucson, AZ 85721 (e-mail: internet: kgreen@ccit.arizona.edu).

-Accepted by previous editor, Charles W. Eriksen during natural conversations, average syllable duration can vary by more than 300 msec across changes in speaking rate (Miller, Grosjean \& Lomanto, 1984), whereas overall durations of $\mathrm{CV}$ syllables uttered in isolation can vary by more than $500 \mathrm{msec}$ (Miller \& Baer, 1983; Miller et al., 1986). Acoustic cues that are temporal in nature, such as voice onset time (VOT), transition duration, or frication duration, are similarly affected (Miller \& Baer, 1983; Miller et al., 1986; Summerfield, 1975). This variation creates a problem for the perceptual system, because it results in a mapping relationship between signal and phonetic percept that is exceedingly complex.

One temporal characteristic that has been extensively studied with respect to changes in speaking rate is voice onset time (VOT). Studies of the production of voiced and voiceless stop consonants indicate that voiced stops are typically produced with shorter VOT values than voiceless stops are (Lisker \& Abramson, 1964). However, a change in speaking rate can result in a considerable shift in the distribution of VOT values, especially for voiceless stops. For example, Miller et al. (1986) examined the VOT values of the stop consonants $/ \mathrm{bi} /$ and 
/pi/ produced at different rates of speech varying from slow to fast. Their study revealed a consistent shift in the VOT distributions for both consonants as a function of syllable duration, which varied systematically with speaking rate. As rate increased (and syllable duration decreased), the VOT distributions shifted toward shorter VOT values. When rate decreased (and syllable duration increased), there was a shift in the VOT distributions toward longer VOT values. Such shifts occurred for both the voiced and voiceless tokens, although they were typically larger for the voiceless tokens. The result is that at fast rates of speech, there was considerable overlap in the VOT distributions for the voiced and voiceless tokens (see also Summerfield, 1975); that is, a large number of voiceless tokens are produced with VOT values that are shorter than those for many voiced tokens. Volaitis and Miller (1992) have shown that a similar situation exists for alveolar and velar consonants as well. This situation raises the question of how the perceptual system is able to make accurate voicing decisions, especially when faced with tokens produced at fast rates of speech.

Studies of speech perception indicate that the perceptual system maintains accurate categorization by adjusting the criterion VOT value used to make the voicing decision, with respect to speaking rate. For example, Green and Miller (1985) demonstrated that the overall duration of a target syllable, which varies as a function of speaking rate, influences the voicing decision of the syllable's initial stop consonant as either $/ \mathrm{b} /$ or $/ \mathrm{p} /$. Green and Miller created a speech continuum ranging from $/ \mathrm{bi} /$ to $/ \mathrm{pi} /$ by editing natural speech tokens. Each member of the continuum had an overall syllable duration of $302 \mathrm{msec}$. Two additional continua were created by removing the final 92 or $141 \mathrm{msec}$ of vowel from each stimulus in the original series. These stimuli were then presented to listeners who were asked to identify each stimulus as either " $b$ " or " $p$," and to judge its speaking rate along a 10-point scale. Green and Miller found reliable shifts in judged speaking rate as a function of overall syllable duration. As the overall duration of the target syllables decreased, the judged speaking rate of the syllables increased. More important, Green and Miller also obtained reliable shifts in the voicing boundary as a function of syllable duration. With a decrease in overall syllable duration, the voicing boundary shifted toward shorter VOT values (see also Summerfield, 1981). Miller and Volaitis have shown that the effect of syllable duration on the voicing decision is not limited to tokens with VOT values located near the category boundary. Instead, changes in syllable duration appear to result in a shifting of the entire voiced and voiceless categories, affecting not only boundary tokens, but all the tokens in the category, including those judged as most representative or "prototypical" of the category.

The relationship between syllable duration and voicing boundary is complex, however. Summerfield (1981) has shown that increasing the overall duration of a target syllable by adding acoustic information corresponding to another phonetic segment (as in $/ \mathrm{biz} /-/$ piz/) can result in a shift in the voicing boundary toward shorter VOT values. According to Miller and Liberman (1979), who obtained a similar finding with respect to the stop-semivowel distinction of $/ \mathrm{bad} / \mathrm{versus} / \mathrm{wad} /$, lengthening overall syllable duration by adding a third segment at the end of the syllable actually specifies a faster articulation rate to the perceptual system because there are three phonetic segments for a given syllable duration, rather than just two. Miller and Liberman proposed that articulation rate is not calculated solely on the basis of overall syllable duration, but rather on the number of phonetic segments for a specified syllable duration. Thus, overall syllable duration is used by the perceptual system to calculate the articulation rate of a given syllable, which then influences the phonetic categorization of an initial consonant.

Rate information extracted from speech outside of the relevant target syllable also influences the voicing decision. For example, the rate at which a precursor phrase is spoken influences the voicing decision of a target syllable presented at the end of the phrase. When the rate of the precursor phrase is fast, the voicing boundary shifts toward shorter VOT values. When the precursor phrase is spoken slowly, the voicing boundary shifts toward longer VOT values (Diehl, Souther, \& Convis, 1980; Gordon, 1988; Kidd, 1989; Miller, Green, \& Schermer, 1984; Summerfield, 1981). However, Summerfield (1981) has shown that temporal continuity between the precursor context and target syllable is important. If the precursor context is separated from the target syllable by $500-1,000 \mathrm{msec}$ of silence, it has little influence on the voicing decision.

A precursor phrase can therefore provide a rate context that can be used to normalize the VOT information on which the voicing decision of a target syllable is based, as long as there is temporal continuity between the precursor phrase and the target syllable. However, temporal continuity is not the only important relationship between the precursor phrase and the target syllable. There must be continuity with respect to the talker as well. Diehl et al. (1980) have shown that a change in talker between the precursor phrase and the target syllable prevents the precursor phrase from providing rate information about the target syllable. In a follow-up set of experiments, Diehl et al. investigated whether the absence of a rate effect by the precursor phrase was the result of differences in fundamental frequency, the change in formant frequencies that implied a change in vocal tract sizes between the precursor phrase and the target syllable, or both. Their results showed that changing either the fundamental frequency or the implied vocal tract size reduced or eliminated the rate-normalization effect of the precursor phrase.

Thus, for rate information that is extrinsic to the target item (as in a precursor phrase), both temporal continuity and talker continuity are necessary for rate normalization to occur. However, with regard to rate information that is intrinsic to the target item (as in overall syllable duration), it is currently unknown what types of continuity, either temporal or talker, are necessary for rate normalization. The purpose of the current set of experiments was to investigate this question with respect to one type of continuity, that of the talker. There are two reasons why it 
is of interest to examine whether rate normalization of information within a syllable is sensitive to changes in talker continuity. The first is that a single target syllable represents a very short period of time over which to calculate information regarding the source characteristics of the talker. It is therefore possible that a change in talker within a syllable might occur so rapidly that the perceptual system would be unable to notice or respond to the change and rate normalization would therefore be unaffected. Such a finding would be different from the findings of Diehl et al. (1980), in which the change in talker characteristics between the precursor phrase and the test utterance did result in a significant decrease in rate normalization. Presumably, this decrease occurred because the perceptual system had ample time to extract the characteristics of the talker during the precursor phrase and to compare such characteristics with those of the test utterance. When the characteristics failed to match, the precursor was no longer used as a context for rate normalization.

A second reason why the question of talker continuity within a syllable is of interest is that it is relevant to the issue of whether rate normalization is the result of a single underlying mechanism, or whether separate mechanisms are required: one that adjusts for variation that is external to the test syllable, and one that is sensitive to variation within the test syllable itself. Summerfield (1981) addressed this issue in a series of studies examining how rate variations in a precursor phrase and the test syllable itself influence the perceived voicing of a $\mathrm{CV}$ syllable. Summerfield (1981) found the effects of a precursor phrase to be small and mostly limited to the local context of the test utterance. Summerfield (1981) proposed that the influence of speaking rate on phonetic perception primarily reflects the recovery of intrinsic timing information contained within the speech signal. In effect, he proposed a single rate mechanism that is responsible for handling changes in speaking rate, regardless of whether the changes occur within a syllable or in the immediately preceding context. A similar notion that a common mechanism is responsible for rate normalization underlies the view of Diehl and his colleagues that rate normalization is the result of a contrast in the durations of local elements in an auditory signal (c.f. Diehl \& Walsh, 1989). Although the views of Summerfield and Diehl differ with respect to the basis of rate normalization-that is, as a speechspecific or general auditory mechanism-both are consistent with a common mechanism accounting for both syllable-external and -internal rate effects.

However, the results of certain studies create difficulties for the notion that a common mechanism is responsible for normalization to both syllable-internal and -external rate variation. For example, even the articulation rate of words that are quite removed from the test syllable can influence the perception of the syllable (Miller, Green, \& Schermer, 1984; Summerfield, 1981). Although such effects are admittedly small, they are highly reliable, occurring even when listeners are not explicitly required to focus on the precursor phrase (Miller, Green, \& Schermer, 1984). More recently, Wayland, Miller, and Volai- tis (1992) have demonstrated that rate information derived from within the syllable itself has a different impact on the structure of the voiceless / $p$ / category than rate information extracted from a precursor sentence. Miller and Volaitis (1989) have shown that a change in syllable rate shifted the best exemplar of the $/ \mathrm{p} /$ category toward longer VOT values and broadened the overall category width. However, a slower precursor phrase apparently only influences the best exemplar of the /p/ category, with no effect on the category width (Wayland et al., 1992). On the basis of these findings, Wayland et al. argue that separate mechanisms are involved in the extraction and use of rate information both within a syllable and external to it. If this proposal is correct, it is possible that the extraction of rate information from within a syllable and from outside of the syllable might be influenced by different aspects of continuity. Therefore, in the present study, we investigated the influence of syllable duration on the perception of VOT when the initial portion of the syllable was produced by one talker and when the remainder of the syllable was produced by a different talker.

\section{EXPERIMENT 1}

In the first experiment, a long $(350-\mathrm{msec}) / \mathrm{bi} /-/ \mathrm{pi} /$ continuum was synthesized with pitch and formant values appropriate for a female talker. A short (118-msec) /bi/-/pi/ continuum was created by removing the final $232 \mathrm{msec}$ of the vowel of each member in the long continuum. A $232-\mathrm{msec} / \mathrm{i} /$ vowel with pitch and formant values appropriate for a male talker was then synthesized and appended to each of the tokens in the short continuum, creating a third hybrid continuum. The tokens from these three continua were presented to listeners for identification as "b" or "p."

If talker continuity across the syllable is important for calculation of overall syllable duration, a change in talker partway through the syllable should disrupt or modify the calculation of speaking rate for the target syllable. This should result in the hybrid tokens' producing a voicing boundary that is similar to that of the short tokens. Alternatively, if talker continuity across the syllable is not necessary for calculation of overall syllable duration, the hybrid tokens should produce a voicing boundary that is similar to that of the long tokens, because they have the same overall syllable duration.

\section{Method}

Subjects. The subjects were 10 undergraduate sudents at the University of Washington who were either paid or given course credit as an incentive to participate. None of the subjects reported any history of a speech or hearing disorder, and all were native speakers of English.

Materials. The stimuli consisted of three synthetic /bi/-/pi/ speech continua varying in VOT from 10 to $55 \mathrm{msec}$. The first continuum was created with the use of a version of the Klatt software synthesizer (Klatt, 1980) implemented on an LSI-11/73 computer. A series of 10 syllables was synthesized with pitch and formant values appropriate for a female talker. ${ }^{1}$ Each syllable was $350 \mathrm{msec}$ in duration and consisted of a 5 -msec release burst, followed by 
a period of formant transitions ( $30 \mathrm{msec}$ for $F 1 ; 45 \mathrm{msec}$ for $F 2$ and $F 3$ ), and a $290-\mathrm{msec}$ period of steady-state information. The synthesizer was set in cascade mode, and only four formants were used in order to provide a better approximation of a female voice (see Klatt, 1980). The steady-state values (in hertz) of the four formants were $F 1=376, F 2=2508, F 3=3100$, and $F 4=3300$. The bandwidths of the formants (in hertz) were $F 1=50, F 2=$ $100, F 3=130$, and $F 4=250$. The $/ \mathrm{bi} /$ end point of the continuum had starting values for the first three formants (in hertz) of $F 1=$ $290, F 2=1629$, and $F 3=2450(F 4$ remained a constant 3300 across the entire utterance). Voicing began $10 \mathrm{msec}$ after the onset of the release burst plus $5 \mathrm{msec}$ of aspiration. The aspiration was created by stimulating the formants with a noise excitation source and widening the $F 1, F 2$, and $F 3$ formant bandwidths to 450,170 , and $200 \mathrm{~Hz}$, respectively. The fundarnental frequency $(F 0)$ started at $180 \mathrm{~Hz}$, rose linearly over the first $50 \mathrm{msec}$ to $206 \mathrm{~Hz}$, and then fell linearly over the remaining $300 \mathrm{msec}$ to $160 \mathrm{~Hz}$. To make the remainder of the continuum, the time from the onset of the release burst to the onset of voicing was increased by increasing the duration of aspiration in 5-msec increments. This had the effect of increasing both the $F 0$ and $F 1$ onset frequency at the time of voicing, across the continuum. We refer to the members of this continuum as the long tokens.

A second continuum was made by using a waveform editing program to remove the final $232 \mathrm{msec}$ of the vowel portion of each member of the original continuum. The tokens in this second continuum were therefore identical to those in the first, except for their overall syliable duration of $118 \mathrm{msec}$. We refer to the members of this continuum as the short tokens.

Next, a single /i/ vowel, $350 \mathrm{msec}$ in duration and with pitch and formant values appropriate to a male speaker, was synthesized. ${ }^{2}$ The frequency values (in hertz) of the formants were $F 1=330$, $F 2=2200, F 3=3000$, and $F 4=3300$. The bandwidths (in hertz) of the formants were $F 1=50, F 2=100, F 3=130$, and $F 4=$ 250 . The fundamental frequency of the vowel rose linearly from 100 to $120 \mathrm{~Hz}$ over the first $50 \mathrm{msec}$, with a linear decline to $90 \mathrm{~Hz}$ over the remaining $300 \mathrm{msec}$. By using the waveform editor, the final $232 \mathrm{msec}$ was excised from the vowel and appended to the end of each of the short tokens. This resulted in a third, hybrid, continuum, in which the initial $118 \mathrm{msec}$ of each token were identical to the duration of the short stimuli, and the overall syllable duration was equal to that of the original long stimuli. Thus, the initial $118 \mathrm{msec}$ of each token corresponded to the female voice, and the remaining $232 \mathrm{msec}$ corresponded to the male voice.

Procedure. Each subject participated in a single 40-min session. During the first half of the experiment, the subject was presented with 21 different randomized orders of the short and long tokens. The first order was considered practice and was excluded from the analysis. Thus, 20 responses to each stimulus were collected. In the second half of the experiment, the subject was presented with 31 different randomized orders of the hybrid tokens. Again, the first order was considered practice and not scored, leaving a total of 30 responses to each stimulus. In both halves of the experiment, the subject was simply asked to identify each stimulus as either " $b$ ", or "p."

The subjects were tested individually in a small, sound-attenuated room. The stimuli were presented on line, using a lab computer (DEC LSI-11/73). The stimuli were output at a 10-kHz sampling rate, low-pass filtered at $4.9 \mathrm{kHz}$, amplified (Yamaha $A-420$ stereo amplifier), and presented to subjects over headphones (Telephonics TDH-39P) at a comfortable listening level (approximately $78 \mathrm{~dB}$ SPL). The subjects indicated their responses by pressing the appropriately labeled button on a response pad located in front of them. This pad was actually a two-button Microsoft mouse hooked up to a computer terminal (NDS GP-29) and could comfortably fit under the palm of the subject's hand. The lab computer recorded all responses and controlled the rate of presentation. The stimuli were presented every $2 \mathrm{sec}$.

\section{Results}

The identification results for the short, long, and hybrid continua are presented in Figure 1. Consider first the functions for the short and long stimuli. Consistent with the results of previous studies, there is a shift in the identification function toward longer VOT values for the long tokens than for the short. Next consider the function for the hybrid tokens. As shown in the figure, this function is nearly identical to that of the short stimuli. The mean VOT boundaries for the three continua provide a measure of the difference in their identification functions. The individual voicing boundaries were determined by fitting a linear regression line to the data in the boundary region of each individual identification function, taking as the category boundary the VOT value that corresponded to $50 \% / \mathrm{b} /$ responses. The mean boundaries were 21.2 , 24.3 , and $20.8 \mathrm{msec}$ for the short, long, and hybrid continua, respectively. These boundaries were analyzed by means of a one-way analysis of variance (ANOVA) with auditory continuum as the single factor. The results of the ANOVA revealed a significant effect of auditory continuum $[F(2,18)=13.7, p<.0005]$. An analysis of the separate mean boundaries indicated there was no significant difference between the short and the hybrid continua $(p>.05)$, although there were significant differences between the short and the long $(p<.01)$ as well as the hybrid and the long continua $(p<.01)^{3}$

In summary, the voicing boundary for the hybrid continuum was similar to that of the short stimuli, even though the overall syllable duration of the hybrid stimuli was equal to that of the long stimuli. This result demonstrates that a change in the talker characteristics of the vowel disrupts the calculation of rate information across the entire syllable even when the overall phonetic quality of the vowel is maintained. What is not clear from this experiment is what aspects of the source characteristicsfundamental frequency or vowel formant values-need to

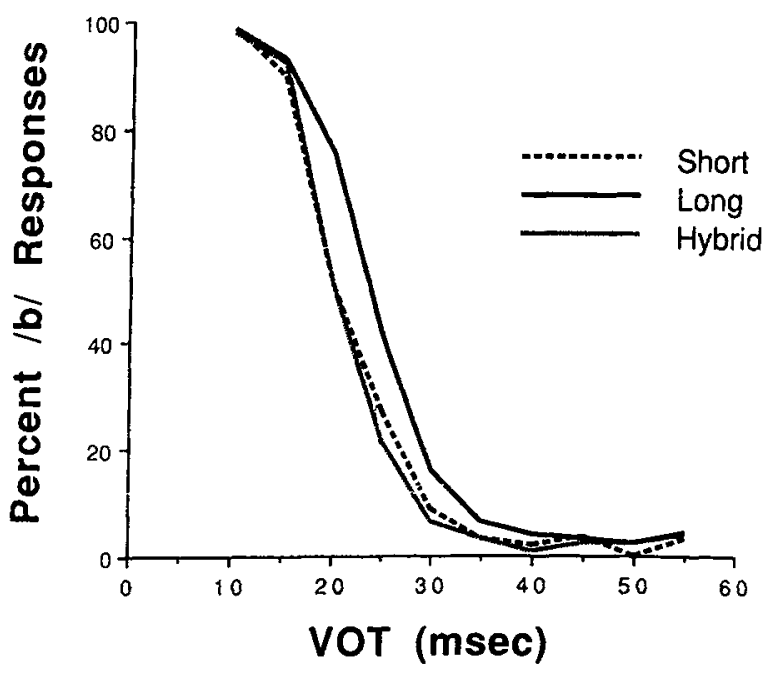

Figure 1. Percentage of /b/ responses for the short, long, and hybrid tokens (Experiment 1). 
be continuous across the entire syllable. In Experiment 1, both of these variables were changed between the first and second halves of the syllable. In Experiment 2, these two variables were manipulated independently in order to determine the contribution of each variable separately.

\section{EXPERIMENT 2}

The purpose of the second experiment was to determine whether a change in either the fundamental frequency or the formant structure would be sufficient to influence the integration of duration information by independently manipulating these two talker characteristics. Two new /i/ vowels were synthesized. The first had the pitch appropriate to a male talker and formants appropriate to a female talker. The second vowel had the pitch appropriate to a female talker and the formants appropriate to a male talker. These new vowels were appended to each of the tokens in the short continuum, creating two new hybrid continua, hybrid A and hybrid B. These new continua, along with the original short and long continua, were presented to listeners for identification.

If continuity in both fundamental frequency and vowel formant structure are necessary across the entire syllable for integration of duration information, we would expect to find that both sets of hybrid tokens produce voicing boundaries similar to those of the original short stimuli, as in Experiment 1. This pattern of results would be similar to that obtained by Diehl et al. (1980) for the influence of a precursor phrase on a target syllable. Alternatively, it is possible that neither variable by itself is capable of signaling a change in talker when that change occurs within the test syllable. Such a situation might occur simply because the change in talker signaled by each variable separately might be so subtle that the perceptual system does not have a chance to notice it over the short duration of the syllable. If so, both sets of hybrid tokens ought to produce VOT boundaries similar to those of the original long stimuli. Finally, it might be the case that only one of the two variables is necessary for calculating rate information from within a syllable. This situation would result in an asymmetry between the two hybrid continua, with one continuum producing voicing boundaries similar to those of the short tokens, and the other producing voicing boundaries similar to those of the long.

\section{Method}

Subjects. The subjects were 20 new undergraduate students at the University of Washington. Each was either paid or given course credit as an incentive to participate in the experiment. All the subjects were native speakers of English, and no one reported any history of a speech or hearing disorder.

Materials. The stimuli consisted of four synthetic $/ \mathrm{bi} /-/ \mathrm{pi} / \mathrm{con}$ tinua varying in VOT from 10 to $55 \mathrm{msec}$. Two of the continua consisted of the original short and long tokens from Experiment 1. To make the remaining two hybrid continua, two new /i/ vowels, $350 \mathrm{msec}$ in duration, were synthesized. The first vowel had formant frequency values and bandwidths identical to the steady-state values of the female tokens used in Experiment 1. The fundamental frequency contour for this vowel was identical to that of the male /i/ vowel created in Experiment 1. A waveform editing program was used to excise the final $232 \mathrm{msec}$ of the vowel, which were appended to each of the short tokens, creating a new set of hybrid stimuli: hybrid A (formant-constant) tokens.

A second vowel with formant frequency values and bandwidths identical to the values from the male / $i$ / vowel created in Experiment 1 was also synthesized. However, the fundamental frequency contour for this second vowel was identical to that of the long female stimuli created in Experiment 1. Again, using the waveform editing program, the final $232 \mathrm{msec}$ of each vowel was excised and appended to each of the short tokens creating a second set of hybrid stimuli: hybrid B (fundamental-constant) tokens.

Procedure. The procedures and equipment were essentially the same as those used in Experiment 1 except for the following differences. Half the subjects were assigned to one experimental group (group A) while the other half were assigned to a different group (group B). The subjects in group A were presented with the short and long tokens in the first half of the experiment and the hybrid $A$ tokens in the second half. The subjects in group B were also presented with the normal short and long tokens, followed by the hybrid $\mathrm{B}$ tokens.

\section{Results}

The identification results for the three continua presented to subjects in group A are presented in Figure 2. As can be seen in the figure, the overall pattern of responses for these data is very similar to that found in Experiment 1. Again, there is a shift toward longer VOT values in the identification function of the long tokens as opposed to the short. More importantly, the hybrid A tokens, which maintained continuity in the formant frequency values across the entire vowel while abruptly changing the pitch, produced an identification function that was similar to that for the short tokens. The mean VOT boundaries for the three continua were 25.0,28.5, and 24.9 for the short, long, and hybrid $A$ tokens, respectively. A one-way ANOVA of the boundary values for each subject was significant $[F(2,18)=14.5, p<.0005]$. Post hoc analysis of the mean boundaries indicated no sig-

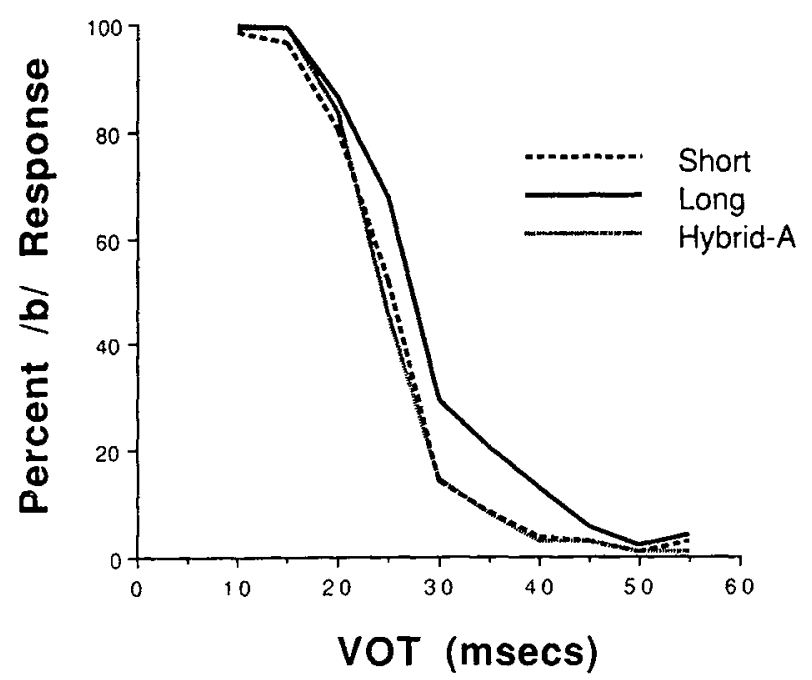

Figure 2. Percentage of $/ b /$ responses for the short, long, and hybrid A (formant-constant) tokens (Experiment 2). 


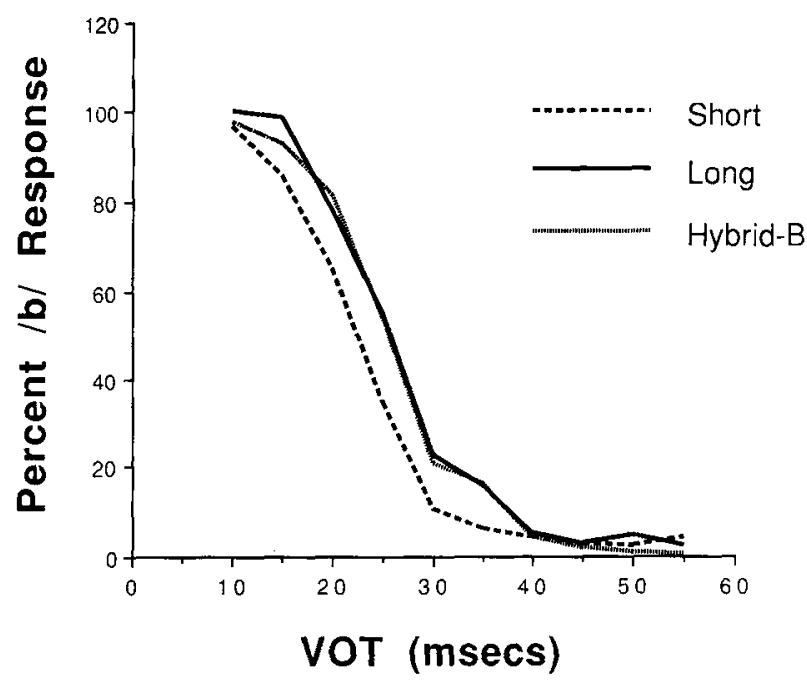

Figure 3. Percentage of $/ b /$ responses for the short, long, and hybrid B (fundamental-constant) tokens (Experiment 2).

nificant difference between the short and the hybrid A tokens $(p>.05)$, although there were significant differences between the short and the long $(p<.01)$ and the hybrid A and the long tokens $(p<.01)$.

The results for the subjects in group B are presented in Figure 3. Here there is a different pattern of responses from that of Experiment 1. As in the first experiment, there is again a shift toward longer VOT values in the identification function of the long tokens as opposed to the short. More important, the hybrid B tokens, which maintained pitch continuity across the entire syllable while abruptly changing the formant values in the middle of the vowel, produced an identification function that was nearly identical to that of the long tokens. The individual mean boundaries $(22.4,26.6$, and 25.8 for the short, long, and hybrid B tokens, respectively) were again analyzed in a one-way ANOVA $[F(2,18)=19.4, p<.0001]$. Post hoc analysis indicated a significant difference between the short and long tokens $(p<.01)$. However, for these stimuli, there was also a significant difference between the short and hybrid B tokens $(p<.01)$, and no significant difference between the long and hybrid B tokens.

The results of this experiment indicate that an abrupt discontinuity with respect to fundamental frequency is enough to disrupt the calculation of duration across an entire syllable. Therefore, the perceptual system is sensitive to a change in talker within a syllable even when that change is signaled by just one variable, a change in pitch. Moreover, this finding is similar to that of other studies that have demonstrated the importance of fundamental frequency in maintaining the phonetic continuity of the acoustic signal. For example, pitch continuity has been shown to be important in grouping together both successive formant patterns for the perception of consonants (Darwin \& Bethell-Fox, 1977) and simultaneously presented formants (Darwin, 1981). The results of this sec- ond experiment demonstrate that pitch continuity is also necessary for the calculation of speaking rate within a target syllable.

This finding contrasts with earlier findings of Diehl et al. (1980), who used a precursor phrase. They found that a change in either fundamental frequency or formant frequency values between a precursor phrase and a target syllable would reduce or eliminate the influence of the rate of the precursor phrase on the identification of the target. In the present experiment, continuity in the formant values across the entire syllable was not necessary to maintain duration and therefore rate continuity. One possible explanation of the difference in the results of the present study may be that when one is normalizing for speaking rate within a syllable, only pitch continuity is necessary across the entire syllable for the calculation of syllable duration. An alternative possibility is that the amount of change in the formant frequency values used in Experiment 2 was not enough to reduce or eliminate the integration of duration information across the syllable. In the present study, the ratio of the female to male formant frequencies was 1.14 (for $F 1$ and $F 2$ ), whereas Diehl et al. used a ratio of 1.19 . It was possible that a greater change in formant frequency values might disrupt the calculation of duration across the syllable. This possibility was explored in a third experiment.

\section{EXPERIMENT 3}

The purpose of the third experiment was to determine whether a greater change in the formant frequency values would inhibit the integration of duration information across a syllable with a constant pitch continuity. To address this question, a new male /i/ vowel was synthesized with even lower formant frequency values than those of the male vowel used in Experiment 1. The ratio of the female to male formant values for this new /i/ vowel was 1.2 , which is the ratio that occurs for female to male speakers saying the /i/ vowel in Peterson and Barney (1952). However, the fundamental frequency used was the same as that of the male vowel in Experiment 1. A second / i/ vowel was also synthesized by using the lower formant frequency values but with a fundamental frequency identical to those of the original female tokens. These /i/ vowels were then appended to the short tokens to create two new hybrid continua: hybrid $C$ and hybrid $D$ tokens.

\section{Method}

Subjects. The subjects were 16 new undergraduate students at the University of Washington who were paid or given course credit as an incentive to participate. None of the subjects reported any history of a speech or hearing disorder, and all the subjects were native speakers of English.

Materials. The stimuli consisted of four synthetic $/ \mathrm{bi} /-/ \mathrm{pi} / \mathrm{con}$ tinua varying in VOT from 10 to $55 \mathrm{msec}$. Two of the continua consisted of the original short and long tokens from Experiment 1. To make the remaining two hybrid continua, two new /i/ vowels $350 \mathrm{msec}$ in duration were synthesized. The formant frequency values (in hertz) for both vowels were $F 1=313, F 2=2090, F 3=$ 2700 , and $F 4=3300$. The bandwidths of the formants were the 
same as those used for the original male vowel in Experiment 1. The fundamental frequency contour for the first / $i$ / vowel was identical to that used in the original male vowel in Experiment 1. The final $232 \mathrm{msec}$ of this vowel was appended to each of the short tokens, creating a new hybrid $\mathrm{C}$ continuum, which, like the original hybrid continuum used in Experiment 1, changed from a female talker to a male talker but with lower formant frequency values. The fundamental frequency contour of the second /i/ vowel was identical to that of the original female tokens. The final $232 \mathrm{msec}$ of this vowel were also appended to each of the short tokens, creating a new hybrid D continuum, which, like the hybrid $B$ tokens used in Experiment 2, maintained pitch continuity across the syllable but with a greater change in the formant frequency values.

Procedure. The procedures and equipment were the same as those used in Experiment 2. The subjects in group A were presented with the normal short and long tokens in the first half of the experiment and the hybrid $\mathrm{C}$ tokens in the second half. The subjects in group $\mathrm{B}$ were also presented with the normal short and long tokens, followed by the hybrid $\mathrm{D}$ tokens.

\section{Results}

The identification results for the three continua presented to the group A subjects are displayed in Figure 4. As shown in the figure, the overall pattern of responses is very similar to that found in Experiment 1. This is a shift toward longer VOT values in the identification function of the long tokens as opposed to the shorts. Moreover, there is little difference in the identification functions for the short and the hybrid $\mathrm{C}$ tokens. An analysis of the mean VOT values $(24.1,27.9$, and 23.5 for the short, long, and hybrid $\mathrm{C}$ tokens, respectively) again demonstrated a significant effect of auditory continuum $[F(2,14)=8.3, p<.005]$. Post hoc analysis revealed no significant difference between the short and hybrid $\mathrm{C}$ tokens $(p>.05)$, although there were significant differences between the short and long $(p<.01)$ and the hybrid $\mathrm{C}$ and long $(p<.01)$ continua.

The results for the subjects in group B are presented in Figure 5. As in the previous experiments, there is the

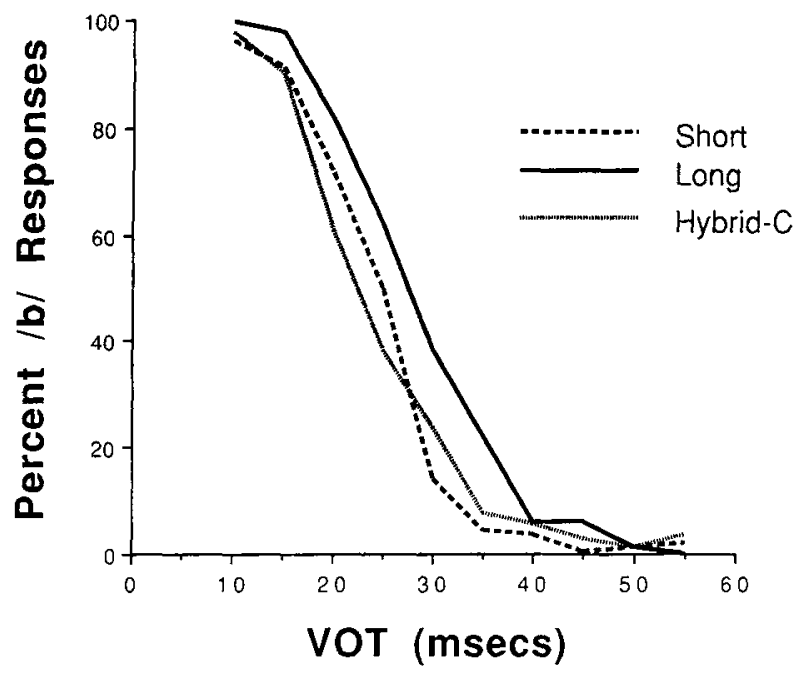

Figure 4. Percentage of $/ b /$ responses for the short, long, and hybrid $\mathrm{C}$ tokens (Experiment 3 ).

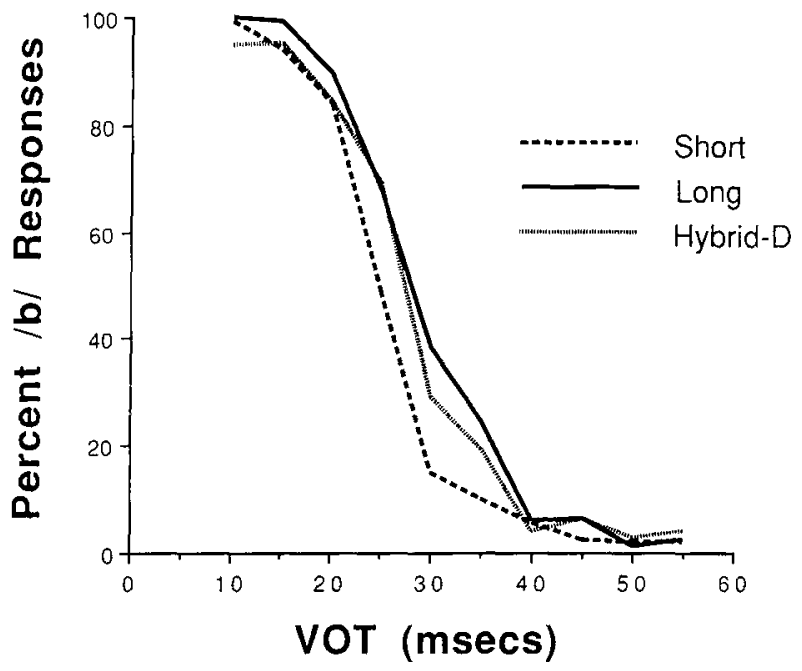

Figure 5. Percentage of $/ b /$ responses for the short, long, and hybrid D tokens (Experiment 3).

shift in the identification function toward longer VOT values for the long tokens as opposed to the short tokens. However, like the hybrid B tokens in Experiment 2, which also maintained pitch continuity while abruptly changing the formant values in the middle of the vowel, the hybrid D tokens produced an identification function that was very similar to that for the long tokens. An analysis of the mean VOT boundaries $(25.2,28.9$, and $27.8 \mathrm{msec}$ for the short, long, and hybrid $\mathrm{D}$ tokens, respectively) again indicated a significant effect of auditory continuum $[F(2,14)=6.8$, $p<.01]$. Post hoc analyses again indicated a significant difference between the short and long continua $(p<.01)$, as well as the short and hybrid D tokens $(p<.05)$. However, there was no significant difference between the long and hybrid D tokens $(p>.05)$.

In summary, the results of this third experiment provide a replication of the finding in Experiment 1 that a change of talker in the middle of a vowel disrupts the calculation of duration across the entire syllable. More importantly, the results of this experiment provide additional support for the notion that calculation of duration and rate information across a syllable will occur despite abrupt and extensive changes in the formant frequency values, as long as pitch continuity is maintained across the syllable.

However, it was still possible that the difference between the pitch-constant and the formant-constant tokens in Experiments 2 and 3 might have been due to an inability in the listeners to actually "detect" a switch in talker when that switch was signaled by a change in just the formant frequencies in the vowel. That is, the change in pitch may have been readily detectable, while the change in formants was not. After listening to the different tokens, the experimenters indicated that this was not the case. It was possible, however, that naive listeners might evaluate the tokens differently during the course of the experiment. This possibility was investigated in a fourth experiment. 


\section{EXPERIMENT 4}

The purpose of the last experiment was to investigate whether listeners could detect a switch in talker in hybrid tokens that maintained pitch continuity and only varied in the formant values. In order to address this issue, the end points of the $/ \mathrm{bi} /-/ \mathrm{pi} /$ continua used in the first three experiments were presented to listeners who judged whether each syllable was produced by one talker or by two different talkers. If listeners could not detect the switch in talker when only the formant values changed from those of a female to those of a male talker, these tokens should receive mostly one-talker judgments. Alternatively, while it might be possible for listeners to detect a switch in talker when just the formant frequencies changed, it might be more difficult than when the pitch changed. This difficulty might only be reflected in the overall time it would take listeners to decide whether the tokens were produced by a single talker or by two different talkers. Therefore, the listeners' response times were also collected and analyzed.

\section{Method}

Subjects. The subjects were 10 new undergraduate students at the University of Arizona who were paid or given course credit as an incentive to participate. All the subjects were native speakers of English, and none reported any history of a speech or hearing disorder.

Materials. The stimuli consisted of the / $\mathrm{bi} /$ and /pi/ end points from the continua used in the earlier experiments. These continua consisted of intact female tokens (Experiment 1); hybrid femalemale tokens (Experiment 1); hybrid female-male pitch/female formants (Experiment 2); hybrid female-female pitch/male formants (Experiment 2); and the hybrid female-female pitch/extreme male formants (Experiment 3). In addition, one additional set of intact tokens was synthesized. These tokens consisted of $/ \mathrm{bi} /$ and $/ \mathrm{pi} / \mathrm{syn}-$ thesized with the female pitch and male formant values used to make the one set of hybrid tokens in Experiment 3. These tokens were added to increase the number and variability of the intact tokens such that they were more similar to the hybrids.

Each of the two sets of intact tokens was presented a total of 20 times while each of the four sets of hybrid tokens was presented 10 times for a total of 160 trials. Half the trials consisted of intact (one-talker) tokens and half consisted of hybrid (two-talker) tokens. The 160 tokens were randomized prior to presentation to the listeners.

Procedure. Each subject participated in a single, 15-min session. The subjects were instructed to listen to each syllable and decide whether the token was produced by a single talker or two different talkers, as quickly as possible. In order to provide some experience with the task, each subject was presented with 16 practice trials prior to the actual collection of data.

The subjects were tested individually in a small, sound-attenuated room. The stimuli were first digitally transferred to a lab microcomputer (NEC 386-20) and then presented on line to the subjects. The stimuli were output at a $10-\mathbf{k H z}$ sampling rate, low-pass filtered at $4.9 \mathrm{kHz}$, amplified (Yamaha AX-630 stereo amplifier), and presented over headphones (Sennheiser HD 450) at a comfortable listening level (approximately $75 \mathrm{~dB}$ SPL). Each subject indicated his/her responses by pressing the appropriately labeled button on a response box located in front of them. The lab computer recorded all responses and controlled the rate of presentation. The stimuli were presented at $1.5-\mathrm{sec}$ intervals.

\section{Results}

Table 1 presents the average percent one-talker judgments in response to the six different types of tokens that were presented to the subjects. As shown in the table, the two sets of intact tokens synthesized either as normal female or with female pitch but male characteristics were judged by listeners to have been produced by a single talker. In contrast, all four sets of hybrid tokens received very few one-talker judgments, indicating that listeners considered these tokens to have been produced by two different talkers. A one-way ANOVA conducted on these data revealed a highly significant effect for group $[F(5,45)=$ $258.07, p<.0001]$. Post hoc analyses indicated that each of the intact tokens was significantly different from each of the hybrid tokens $(p<.01)$. No other significant differences among the means was detected. These data indicate that listeners could easily hear the change in talker characteristics across vowels when that change was signaled by both a change in pitch and formant frequencies from those appropriate for a female talker to those of a male talker. In addition, listeners could also hear the change in talker characteristics when either the pitch or the formant frequencies alone were changed. More importantly, the data indicate that there was no significant difference in the way in which subjects classified the pitch-change or the formant-change tokens. Both sets of tokens produced equivalent one-talker judgments.

It is possible that a difficulty in hearing the change in talker characteristics across the vowel might require a more sensitive measure than the overt one-talker judgments. We therefore examined the listeners' response times to classify the token as well. The average response times to each set of tokens, regardless of whether subjects classified the tokens as spoken by one or two talkers, are presented in Table 1. As shown in the table, there is very little difference in the response times across the six different sets of tokens. A one-way ANOVA of the average response times across subjects revealed a significant effect for group $[F(5,45)=2.8, p<.03]$. Post hoc analysis indicated a significant difference between the hybrid tokens made with the male pitch/female formants and the intact female tokens $(p<.05)$. All other differences among the means were insignificant. These data therefore indicate no difference in the overall discriminability of

Table 1

Average Percent "One-Talker" Judgments (in Milliseconds) for the Six Different Token Types: Experiment 4

\begin{tabular}{|c|c|c|c|c|}
\hline \multirow[b]{2}{*}{ Tokens } & \multicolumn{2}{|c|}{ Vowel Characteristics } & \multirow{2}{*}{$\begin{array}{l}\% \text { One-Talker } \\
\text { Judgment }\end{array}$} & \multirow{2}{*}{$\begin{array}{c}\text { Response } \\
\text { Time }\end{array}$} \\
\hline & Pitch & Formants & & \\
\hline \multirow[t]{2}{*}{ Intact } & female & female & 97 & 671 \\
\hline & female & male & 97 & 695 \\
\hline \multicolumn{5}{|l|}{ Hybrid } \\
\hline \multirow[t]{4}{*}{ Female $\rightarrow$} & male & male & 1.9 & 682 \\
\hline & male & female & 4.5 & 755 \\
\hline & female & male & 3.6 & 703 \\
\hline & female & extreme male & 2.3 & 690 \\
\hline
\end{tabular}


the pitch-change and the formant-change tokens. Otherwise, there would have been a difference in the response times for subjects to judge whether the tokens were produced by one talker or two talkers.

In summary, the results of the fourth experiment indicate that listeners can easily detect the change in talker characteristics when they are signaled by just a change in pitch or just a change in the formant frequencies. This finding is relevant to the findings from Experiments 2 and 3 in which a change in just the pitch prevented the calculation of rate across the entire syllable, whereas a change in just the formant frequencies did not, even though both changes are correlated with a switch in the "source" of the signal from one talker to another. Apparently, the difference between the pitch-change and formant-change tokens with respect to the calculation of speaking rate across the entire syllable is not due to an overall difference with which listeners can detect a change in talkers for the two sets of tokens. The difference appears to lie in the relative importance of the different sources of information for continuity across a syllable. Therefore, unlike in the situation in which a precursor phrase provides an external rate context for the perception of a target syllable, rate information from within the target syllable is calculated across the entire syllable only as long as pitch continuity is maintained.

\section{GENERAL DISCUSSION}

The purpose of the present study was to investigate whether talker continuity within a syllable was necessary for the entire duration of the syllable to be taken into account during phonetic perception. The first experiment demonstrated that talker continuity is necessary for rate normalization to occur within a syllable. This agrees with previous findings that a change in talker characteristics can disrupt the integration of acoustic information during phonetic perception (e.g., Diehl et al., 1980; Darwin \& Bethell-Fox, 1977). The second and third experiments extended the research by demonstrating that continuity of pitch, but not formant structure, appears to be the critical factor in the calculation of rate information across a syllable. The fourth experiment indicated that the lack of an effect of the change in formant structure is not due to an inability in listeners to hear a change in talkers when just the formant frequencies are changed within the test syllable.

These results point out an apparent difference between the type of continuity that is necessary for rate normalization to occur within a syllable and the type of continuity that allows a precursor phrase to serve as the relevant rate context. Recall that Diehl et al. (1980) provided evidence that continuity in both pitch and formant structure are necessary between a precursor phrase and a test syllable in order for the rate of the precursor phrase to influence the phonetic processing of a test syllable. In contrast, the results of the present study indicate that only pitch continuity is necessary for rate information derived from the sylla- ble itself. Thus, there appears to be a distinction in the types of continuity that are required in the two different situations. Continuity in both pitch and formant values is required for rate contexts that precede the syllable of interest, whereas only pitch continuity is necessary for calculating rate information across the syllable itself.

The resuits of this study are relevant to explanations of how the perceptual system adjusts for rate variation during phonetic perception. Currently, there are two important issues that researchers have addressed in this context. The first issue is whether a single mechanism is responsible for handling the variation that occurs externally to the syllable (as in a precursor phrase), as well as variation that occurs internally in the syllable. The alternative possibility is that two separate mechanisms are involved. The second issue concerns the nature of these mechanisms: whether they are specific to speech or are part of our general auditory capacity.

Consider, first, the issue of the number of mechanisms that might be involved in rate normalization during speech perception. Summerfield (1981) addressed this issue in an extensive set of experiments, in which he examined how the perceptual system adjusts to rate variation occurring both in a precursor phrase and in the test utterance itself. Summerfield (1981) described two ways of accounting for his findings. One account consisted of a mechanism that extracted rate information from the relevant context-either the precursor phrase or the syllable itself-and then used that information to adjust or to normalize an internal perceptual clock against which temporally based cues, such as VOT or transition duration, were compared. Summerfield (1981) called this account an extrinsic timing model, because the timing or rate information was extracted separately from the segmental information that it normalized. Moreover, Summerfield's (1981) description of this account required two different mechanisms: one to extract the rate from the precursor phrase, and a separate one to determine the rate of the test syllable. Thus, even though both rate mechanisms influenced the timing of the internal perceptual clock, they responded to different parts of the speech signal and might therefore be affected by different aspects of continuity within the signal.

The second approach described by Summerfield (1981) assumes that timing or rate information is an integral part of the acoustic information used during segmental perception. Therefore, rate information does not have to be separately extracted before being used to normalize perceptual judgments. Instead, higher order properties of the speech signal are extracted and remain invariant over changes in speaking rate. Currently, it is not quite clear exactly what those higher order properties might be, although something such as the ratio of VOT to syllable duration or vowel duration may be involved (Port \& Dalby, 1982).

According to the intrinsic account, higher order properties are extracted from the immediately surrounding phonetic context over an analysis window of approxi- 
mately two to three syllables. One problem for this approach is the effect of the rate of a precursor phrase on phonetic perception. Summerfield (1981), however, argued that such precursor effects were small and primarily limited to the immediately preceding phonetic context, and that notions of extrinsic rate normalization were probably unnecessary. A benefit of this approach is that rate influences from the syllable itself, as well as an external precursor phrase, can be accounted for by the same underlying mechanism.

The results of the present study, when compared with the earlier findings of Diehl et al. (1980), are relevant to this issue, because they indicate that the mechanism that handles rate variation within a syllable and the mechanism that adjusts for rate variation external to the syllable are sensitive to different aspects of continuity across the signal. This difference suggests that two separate mechanisms may be operating: one that adjusts for rate variation within a test syllable, and one that compensates for the variation occurring in an immediately preceding sentence context. This notion is reinforced by recent findings demonstrating that the rate of a precursor phrase has a different impact on the structure of phonetic categories than does the rate of the test syllable by itself (Miller \& Volaitis, 1989; Wayland et al., 1992).

Unfortunately, the results from the present study cannot determine what the nature of these two rate mechanisms might be. It is entirely possible that the mechanism responsible for adjusting to external rate variation operates in an extrinsic normalization manner, whereas the syllable-internal mechanism is sensitive to higher order properties that are intrinsic to the syllable itself. The apparent distinction between the types of continuity that are necessary in the two different situations is consistent with this notion, although further experimentation related to this issue is clearly needed.

The second issue that has been addressed with respect to rate normalization is whether the processes that are involved are specific to speech or are part of our general auditory capacities. For example, Miller and Liberman (1979) maintain that the effect of speaking rate on speech processing is the result of specialized, phonetic operations performed on the speech signal. In their study, Miller and Liberman demonstrated that a stop-semivowel $(/ \mathrm{b} /-/ \mathrm{w} /)$ distinction based on transition duration was influenced by overall syllable duration in a complex manner. Increasing overall syllable duration by simply increasing vowel duration resulted in a shift in the $/ \mathrm{b} /-/ \mathrm{w} /$ category boundary toward longer transition durations, in much the same manner as an increase in vowel duration influenced the $/ b /-/ p /$ boundary in the present study. However, Miller and Liberman found that when syllable duration was increased by the addition of transition information appropriate for a final $/ \mathrm{d} /$ consonant, the $/ \mathrm{b} /-/ \mathrm{w} /$ boundary shifted toward shorter transition durations (see also Summerfield, 1981). This finding led Miller and Liberman to propose that rate effects within a syllable operate through a complex mechanism in which rate information is determined not only by the duration of the syllable, but also by its acoustic and phonetic characteristics. The implication of this proposal is that such a mechanism would be unique to speech signals (although see Fowler, 1990).

An alternative account maintains that at least some of the rate effects on phonetic perception are the result of more general auditory processes that are not specific to speech. With regard to the stop-semivowel contrast, proponents of this view argue that results similar to those of Miller and Liberman (1979) found in studies with human subjects categorizing analogous nonspeech stimuli (Diehl \& Walsh, 1989; Pisoni, Carrell, \& Gans, 1983) and nonhuman subjects (Rhesus macaques) discriminating speech stimuli (Stevens, Kuhl, \& Padden, 1988) suggest that more general processes are at work. In particular, Diehl and his colleagues (Diehl \& Walsh, 1989; Kluender \& Walsh, 1988; Kluender, Diehl, \& Wright, 1988) have proposed that some perceptual effects of changing rate are the result of durational contrast between consonantal and vocalic portions of the signal (although see Fowler, 1990).

Although our findings do not distinguish between these two accounts, they are relevant to such approaches. For example, while consistent with the view of Miller and Liberman (1979) that rate processing is accomplished by phonetic mechanisms, these results also demonstrate that such mechanisms are sensitive to the $F 0$ continuity across the syllable, rather than the spectral continuity. Our findings are also consistent with an auditory account such as that proposed by Diehl (e.g., Diehl \& Walsh, 1989). However, proponents of this view must then explain why overall spectral continuity in a vowel is insufficient to provide suitable contrast for the initial consonantal information unless there is continuity in the fundamental frequency of the vowel as well. In other words, what is it about the nature of contrast phenomena that makes them sensitive to changes in $F 0$ ?

In addition, although Diehl and Walsh (1989) do not explicitly address the issue, their auditory contrast account is consistent with the notion that a single mechanism is responsible for rate normalization regardless of whether the variation occurs prior to the test syllable or within the syllable itself. However, as pointed out above, the results of the present study, combined with the findings of Diehl et al. (1980), indicate that two separate mechanisms are involved. This situation would make an auditory contrast account more complex, because it would have to explain how and why two separate mechanisms are involved in rate normalization. ${ }^{4}$

The results of the present study also raise questions regarding the nature of the mechanism that is responsible for calculating overall syllable duration. For example, several researchers have proposed the existence of a mechanism that is used to track the fundamental frequency of speech signals over time. This information is considered useful for grouping both spectral and temporal aspects of the signal to enhance the recognition of speech presented against noisy or competing backgrounds (Broadbent \& Ladefoged, 1957; Darwin, 1981; Darwin \& Bethell- 
Fox, 1977). Our results are consistent with this notion and indicate that the output of such a mechanism plays a fundamental role in determining the overall duration of a syllable. This duration information would then be combined with information about the phonetic structure of the syllable in order to determine the overall articulation rate of the syllable. Although it is unclear what physiological structures are involved, one possibility is that certain chopper cells located in the ventral cochlear nucleus provide such information through the phase locking of their firing rates to the lower frequencies in the signal (c.f. Greenberg, 1988).

The output of such a mechanism would also be useful for other aspects of speech processing, such as the extraction of talker characteristics, that are used to normalize the speech signal during phonetic processing (Mullennix \& Pisoni, 1990; Mullennix, Pisoni, \& Martin, 1989). Mullennix and Pisoni (1990) have proposed that the subprocesses involved in extracting talker characteristics and phonetic information operate in a parallel contingent fashion. Both processes operate in parallel. However, an output from the phonetic processes is contingent on at least a partial output from the talker-characteristic processes. The results of the present study are consistent with this notion, because they demonstrate that a change in the talker disrupted rate processing. However, we showed that a change in the formant structure of an utterance, although sufficient to change the perception of the talker, was not sufficient to halt rate processing, whereas a change in pitch was sufficient.

Finally, there is an ontogenetic issue. Studies have shown that even young infants are sensitive to the rate characteristics of phonetic segments (Eimas \& Miller, 1980; Miller \& Eimas, 1983). One question raised by the present studies is whether young infants are also sensitive to the talker continuity of an utterance when classifying phonetic segments. If the mechanism underlying rate normalization incorporates some sort of pitch tracker to maintain temporal continuity across an utterance, then, disrupting the pitch characteristics of a syllable should affect infants' categorization of syllables in a manner similar to that demonstrated in the present set of experiments.

In summary, our results agree with previous findings that a change in talker characteristics disrupts the integration of acoustic information during phonetic perception. However, our results extend these findings in an important way by demonstrating that continuity of pitch, rather than formant structure, appears to be the critical factor in the integration of speaking rate within a syllable. Both the pitch change and the formant change were readily discernible, as demonstrated experimentally. However, the change in formant structure, although sufficient to change the perception of the talker who was speaking, was not sufficient to disrupt the perception of rate continuity. A change in pitch was sufficient to disrupt rate continuity. This result raises the possibility that the mechanisms of phonetic perception depend not so much on talker continuity, but on continuity of pitch. Pitch continuity ap- pears to be a prerequisite for extracting rate information during phonetic perception.

\section{REFERENCES}

Broadbent, D. E., \& Ladefoged, P. (1957). On the fusion of sounds reaching different sense organs. Journal of the Acoustical Society of America, 29, 708-710.

DARWIN, C. J. (1981). Perceptual grouping of speech components differing in fundamental frequency and onset-time. Quarterly Journal of Experimental Psychology, 33A, 185-207.

Darwin, C. J., B BeThell-Fox, C. E. (1977). Pitch continuity and speech source attribution. Journal of Experimental Psychology: Human Perception \& Performance, 3, 665-672.

Diehl, R. L., Souther, A. F., \& Convis, C. L. (1980). Conditions on rate normalization in speech perception. Perception \& Psychophysics, 27, 435-443.

Diehl, R. L., \& WALSh, M. A. (1989). An auditory basis for the stimulus-length effect in the perception of stops and glides. Journal of the Acoustical Society of America, 85, 2154-2164.

EImas, P. D., \& MiLler, J. L. (1980). Contextual effects in infant speech perception. Science, 209, 1140-1141.

Fowler, C. A. (1990). Sound-producing sources as objects of perception: Rate normalization and nonspeech perception. Journal of the Acoustical Society of America, 88, 1236-1249.

Gordon, P. C. (1988). Induction of rate-dependent processing by coarsegrained aspects of speech. Perception \& Psychophysics, 43, 137-146.

Green, K. P., \& Miller, J. L. (1985). On the role of visual rate information in phonetic perception. Perception \& Psychophysics, 38, 269-276.

GREENBERG, S. (1988). The ear as a speech analyzer. Journal of Phonetics, 16, 139-149.

KIDD, G. R. (1989). Articulatory-rate context effects in phoneme identification. Journal of Experimental Psychology: Human Perception \& Performance, 15, 736-748.

KLATT, D. K. (1980). Software for a cascade/parallel formant synthesizer. Journal of the Acoustical Society of America, 67, 971-995.

KLuender, K. R., Diehl, R. L., \& WRight, B. W. (1988). Vowellength differences before voiced and voiceless consonants: An auditory explanation. Journal of Phonetics, 16, 153-169.

KLUENDER, K. R., \& WALSH, M. A. (1988). Effect of vowel duration on the perception of syllable-initial $/ \int /$ and $/ \mathrm{t} \mathrm{J} /$. Journal of the Acoustical Society of America, 84 (Suppl. 1), S158.

Lisker, L., \& ABramson, A. S. (1964). A cross-language study of voicing in initial stops. Word, 20, 384-422.

Miller, J. L. (1981). Effects of speaking rate on segmental distinction. In P. D. Eimas \& J. L. Miller (Eds.), Perspectives on the study of speech (pp. 39-74). Hillsdale, NJ: Erlbaum.

Miller, J. L., \& BaER, T. (1983). Some effects of speaking rate on the production of $/ \mathrm{b} /$ and $/ \mathrm{w} /$. Journal of the Acoustical Society of America, 73, 2124-2133.

Miller, J. L., \& Eimas, P. D. (1983). Studies on the categorization of speech by infants. Cognition, 13, 135-165.

Miller, J. L., Green, K. P., \& Reeves, A. (1986). Speaking rate and segments: A look at the relation between speech production and speech perception for the voicing contrast. Phonetica, 43, 106-115.

Miller, J. L., Green, K. [P.], \& Schermer, T. M. (1984). A distinction between the effects of sentential speaking rate and semantic congruity on word identification. Perception \& Psychophysics, 36, 329-337.

Miller, J. L., Grosjean, F., \& Lomanto, C. (1984). Articulation and its variability in spontaneous speech: a reanalysis and some implications. Phonetica, 41, 215-225.

Miller, J. L., \& LibermaN, A. M. (1979). Some effects of lateroccurring information on the perception of stop consonant and semivowel. Perception \& Psychophysics, 25, 457-465.

Miller, J. L., \& Volairis, L. E. (1989). Effect of speaking rate on the perceptual structure of a phonetic category. Perception \& Psychophysics, 46, 505-512.

Mulcennix, J. W., \& Prsoni, D. B. (1990). Stimulus variability and 
processing dependencies in speech perception. Perception \& Psychophysics, 47, 379-390.

Mullennix, J. W., Pisoni, D. B., \& Martin, C. S. (1989). Some effects of talker variability on spoken word recognition. Journal of the Acoustical Society of America, 85, 365-378.

Peterson, G. E., \& Barney, H. L. (1952). Control methods used in a study of the vowels. Journal of the Acoustical Society of America, 24, 175-184.

PISONI, D. B. (1990). Effects of talker variability on speech perception: Implications for current research and theory. In H. Fujisaki (Ed.), Proceedings of the International Conference on Spoken Language Processing (pp. 1399-1407). Tokyo: The Acoustical Society of Japan.

Pisoni, D. B., Carrell, T. D., \& Gans, S. J. (1983). Perception of the duration of rapid spectrum changes in speech and nonspeech signals. Perception \& Psychophysics, 34, 314-322.

PoRT, R. F. (1979). The influence of tempo on stop closure duration as a cue for voicing and place. Journal of Phonetics, 7, 45-56.

PoRT, R. F., \& DAlBY, J. (1982). Consonant/vowel ratio as a cue for voicing in English. Perception \& Psychophysics, 32, 141-152.

Stevens, E. B., Kuhl, P. K., \& Padden, D. M. (1988). Macaques show context effects in speech perception. Journal of the Acoustical Society of America, 84 (Suppl. 1), $\$ 77$.

SUmmerfieLD, A. Q. (1975). How a full account of segmental perception depends on prosody and vice versa. In A. Cohen \& S. G. Nooteboom (Eds.), Structure and process in speech perception (pp. 5168). Berlin: Springer-Verlag.

SUMmerfielD, A. Q. (1981). Articulatory rate and perceptual constancy in phonetic perception. Journal of Experimental Psychology: Human Perception \& Performance, 7, 1074-1095.
Volaitis, L., \& Miller, J. L. (1992). Phonetic prototypes: Influence of place of articulation and speaking rate on the internal structure of voicing categories. Journal of the Acoustical Society of America, 92, 723-735.

Wayland, S. C., Miller, J. L., \& Volaitis, L. E. (1992). The influence of sentence articulation rate on the internal structure of phonetic categories. Journal of the Acoustical Society of America, 92, S2465.

\section{NOTES}

1. The formant frequencies for the /i/ vowel were determined by measuring several tokens of a female talker saying the syllables /bi/ and $/ \mathrm{pi} /$. The frequency values for the first three formants were obtained from the nucleus of the /i/ vowel.

2 . The /i/ formant frequency values were determined by measuring the formant frequencies from a male talker saying the syllables / bi/ and $/ \mathrm{pi} /($ see note 1$)$.

3. All post hoc analyses were performed using the Neuman-Kuels procedure.

4. Of course, other possible explanations for the difference between the present study and that of Diehl et al. (1980) cannot be ruled out. For example, the influence of the speaking rate from a precursor phrase on a test utterance is quite small, and it is possible that the findings of Diehl et al. are a reflection of this (c.f. Summerfield, 1981, note 5).

(Manuscript received October 22, 1992; revision accepted for publication July $21,1993$. 